\title{
CHEMOTHERAPY RESPONSE IN UNDESCENDED (SEMINOMA) TESTIS- A CASE REPORT
}

Anup Kumar, Farrukh Hassan, Surendra Singh, Chandrashekhar Singh

1. Assistant Professor. Department of Radiotherapy, Rajendra Institute of Medical Sciences, Ranchi.

2. Assistant Professor. Department of Surgery, Rajendra Institute of Medical Sciences, Ranchi.

3. Junior Resident. Department of Radiotherapy, Rajendra Institute of Medical Sciences, Ranchi.

4. Junior Resident. Department of Radiotherapy, Rajendra Institute of Medical Sciences, Ranchi.

\section{CORRESPONDING AUTHOR:}

Dr. Anup Kumar,

Asst. Professor, Radiotherapy,

Qt. no. 37, Doctor's colony, Bariatu,

Ranchi- 834009.

E-mail: anupkr_74@yahoo.co.in

Ph: 00919199395419

AIMS: Chemotherapy response in Undescended (Seminoma) testis-a case report

INTRODUCTION: Un descended testis carry a higher potential for malignant transformation than normally descended testis. Seminoma is the most frequent testicular tumor of the testicle in fourth decade of life and constitutes $60-65 \%$ of germ cell neoplasia, several histopathological characteristics have been evaluated and three type of pure seminoma have been described, classic, anaplastic and spermatocytic.

\section{MATERIALS \& METHOD:}

CASE-REPORT: 30 year old man with no previous complain presented with history of progressive enlarging mass and pain abdomen of 6 months duration in June 2008 in Surgery OPD. His CT scan abdomen showed a large well defined soft tissue mass of size $8.4 \times 7.3 \times 7.1 \mathrm{~cm}$ seen in left paracolic space,(pic-1) he underwent laparotomy and en bloc resection of tumor was done. The tumor was completely resected. Histopathology of specimen showed diffuse sheeth of uniformly size round cells with large round vesicular nuclei and abundant pale eosinophilic cytoplasm, thin fibrous septa shows mature lymphocyte infiltrates suggestive of classical seminoma (pic-2). Patient was referred to Oncology OPD in Nov 2008, where USG abdomen and pelvis, X-Ray chest, Tumor markers (alfa fetoprotein, beta HCG \& LDH )and routine blood tests got done, all the tests including tumor markers were within normal limits. Due to non availability of radiation facility in our Institute as well as patient was not keen to undergo radiotherapy and after explaining the pros and cons, he was subsequently planned for two cycle of chemotherapy with Carboplatin $450 \mathrm{mg} / \mathrm{m} 2$ every 4 week. After completion of treatment he was asked to follow-up in Oncology OPD every 3 months. He has completed 3 yrs of follow-up.

DISCUSSION: Surveillance after orchidectomy followed by treatment on relapse is the preferred management option. The overall relapse rate is approximately $15 \%$ to $20 \%$. (1) Recent study failed to validate rete testis invasion as a risk factor for relapse. Size alone is associated with relapse.(1) 
Patients with stage 1 seminoma not treated with adjuvant therapy following inguinal orchidectomy require close follow-up and surveillance is only recommended in patients for whom reliable and committed follow-up is feasible. History and physical examination, serum tumor markers, and CT of the abdomen and pelvis should be performed every 3 to 4 months for the first 3 years, every 6 months from 4 to 7 years, and then annually. Chest x-ray should be obtained every other visit.(2)

RT is highly effective and local control rates approach $100 \%$. However, $80 \%$ of unselected patients are treated unnecessarily, and studies have shown an increased risk of secondary malignancy and cardiovascular toxicity 25 years or more after RT.(1) The standard treatment has been postoperative irradiation of the paraaortic and ipsilateral pelvic nodes so called dog leg radiation therapy. After radiation therapy relapse free survival rates $97 \%$ with a disease free survival of over 99\%.(4) A randomized trial of treatment with either a dog leg (top of T11 through L5, plus ipsilateral iliac nodes to the mid obturator foramen) or para-aortic port (top of T11 to L5 only) showed equal survival with less toxicity in the para-aortic arm.(1)

About $4 \%$ of patients relapse after RT usually in the lungs, posterior mediastinum or supraclavicular nodes and the death rate is under 2\%.(1) A large prospective trial (MRC TE 19/EORTC 30982) comparing adjuvant radiation to one cycle of single-agent carboplatin AUC7 showed no significant difference in relapse, time to relapse and overall survival after a median follow-up of 4 years in stage 1 seminoma. The pattern of relapse differs (more retroperitoneal lymph node relapse with carboplatin versus more pelvic lymph node relapse with radiation), but the more rapid completion of adjuvant therapy and lower incidence of contralateral testicular cancer with carboplatin may be advantageous.(2)

Using two cycle of carboplatin at a dose of $400 \mathrm{mg} / \mathrm{m} 2$,Steiner \& Colleagues reported relapse free survival in 106/108 patients at 60 months.(3)

Reiter \& associates reported $100 \%$ disease free survival in $107 / 107$ patients at 74 month.(3)

CONCLUSIONS: Chemotherapy may be indicated in patient of stage- 1 seminoma where compliance to Radiotherapy is an issue and at the same time it is not associated with much toxicity. Lower incidence of second malignancies with carboplatin may be advantageous.

\section{REFERENCES:}

1. Vincent T. Devita,Jr., Theodore S. Lawrence, Steven A. Rosenberg. Cancer Principles and practice of Oncology. $9^{\text {th }}$ edition. Philadelphia: Lipincott Williams \& Wilkins;2011. Chapter-98, page no 1286-87.

2. Ramaswamy Govindan. The Washington manual of Oncology. 2nd edition. Philadelphia: Lipincott Williams \& Wilkins;2008. Chapter-23, page no 253-54.

3. Hagop M. Kantarjian, Robert A. Wolff, Charles A. Koller. MD ANDERSON Manual of Medical Oncology. New York: Tata McGraw-Hill;2007. Chapter-27, page no 724.

4. Edward C. Halperin, Carlos A. Perez, Luther W. Brady, Rupert K.Schmidt-Ullrich, Principles and practice of Radiation Oncology, $4^{\text {th }}$ Edition. Philadelphia: Lipincott Williams \& Wilkins;2004 Chapter-59, page no 1769-70. 


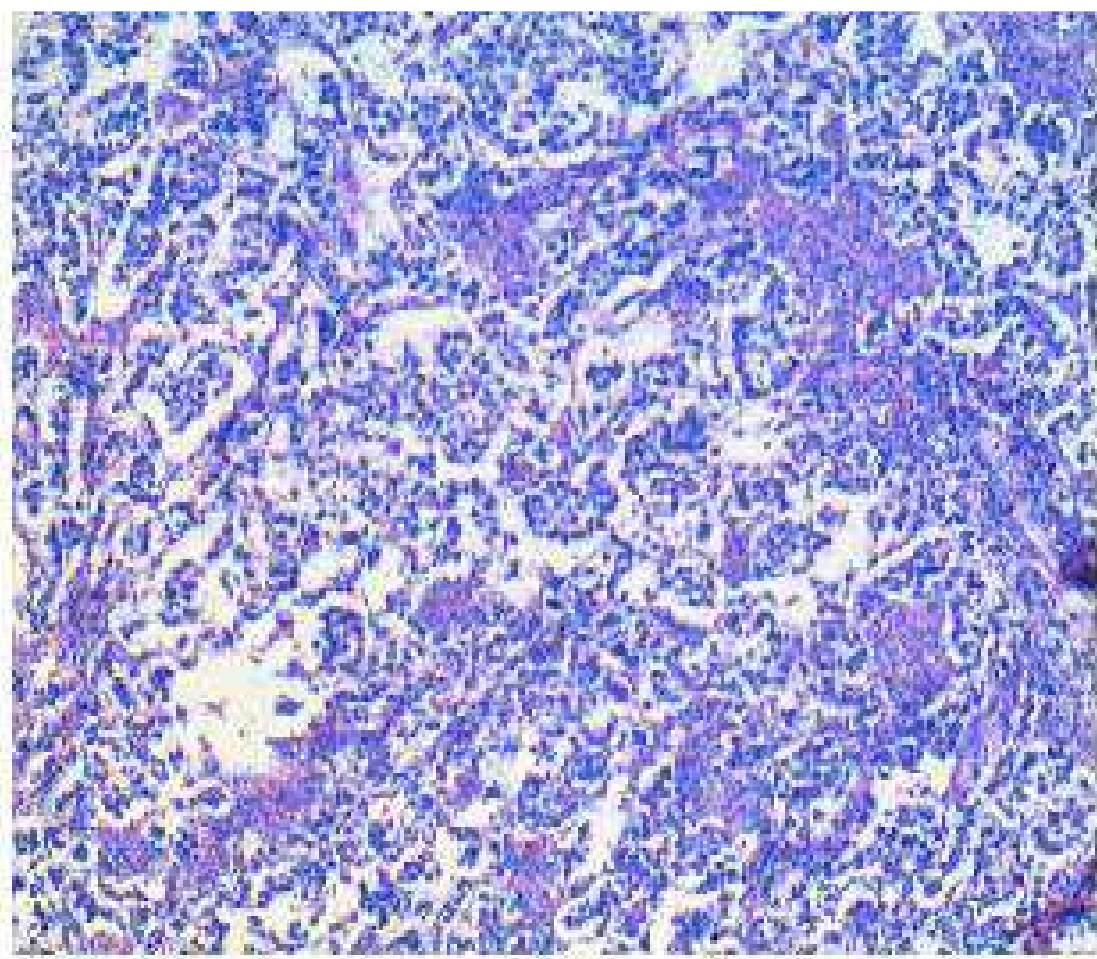

HPE of Specimen Showed-Diffuse sheethe of uniformly size round cells with large round vesicular nuclei and abandant pale eusinophllic cytoplasm thin fibrous septa shows mature lymphocyte infiltrates suggestive of classical seminoma

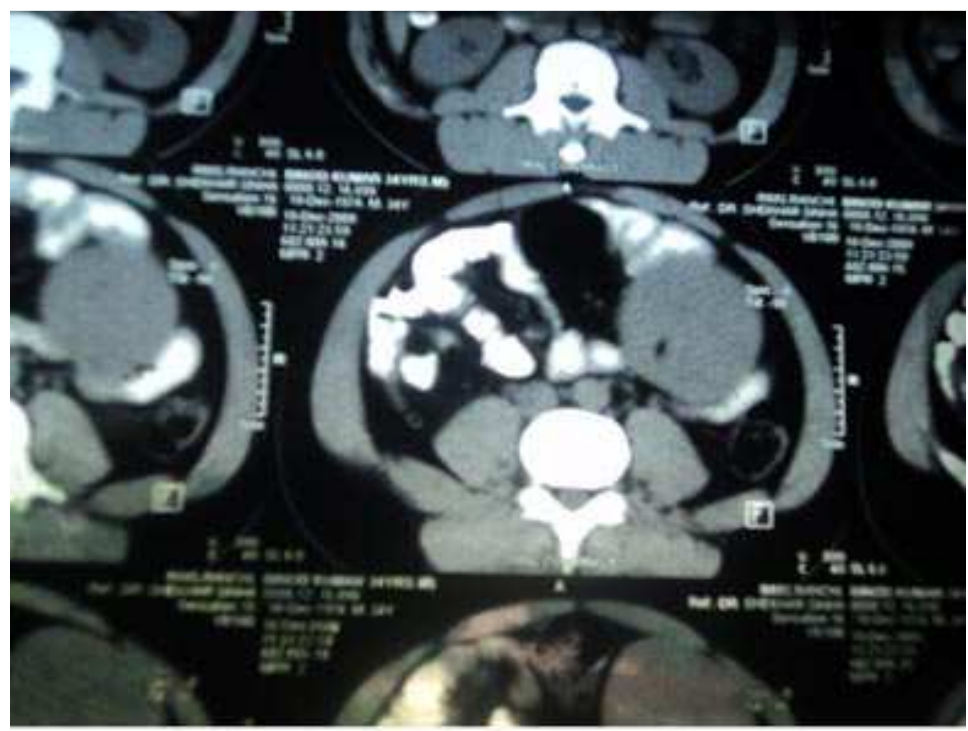

CT Scan abdomen showing a large well defined soft tissue mass of size $8.4,7.3,7.1 \mathrm{~cm}$ in the left paracolic space. 